\title{
Cardiopulmonary Loading and COVID-19: Training Impact during Lockdown among Athletes of Tertiary Learning Institutions in Masvingo, Zimbabwe
}

\author{
Chimonero Prince, PhD \\ Great Zimbabwe University, Zimbabwe \\ Corresponding Mail: pchimonero@gzu.ac.zw
}

\begin{abstract}
Physiological, psycho-social and emotional parameters are critical skill and technical performance motifs in sports endeavors. Despite their noteworthy basis for high-quality athletic efficiencies, training and psycho-social deficiencies are inevitable challenges for athletes in competitive locales. This prospective cohort study adopted the quantitative approach. Its population was 200 participants with a sample of 25 coaches and 50 players drawn from selected tertiary institution teams in Masvingo Province. Study participants were drawn using stratified random sampling. Its thrust was to establish the impact of COVID-19 for return-to-sport athletes during lockdown restrictions. Further, it explored cardiological implications of cardiopulmonary loading parameters among athletes with Severe Acute Respiratory Syndrome-oriented pulmonary ailments and non-diseased group. An adapted updated version of the Oslo Sports Trauma Research Centre questionnaire was used for data collection. Emerging findings revealed substantial disruption of training normalcy from lockdown restrictions. Players risked injury sustenance from solitude unsupervised workouts. Further, high cardiopulmonary loading intensities compromised health conditions of athletes with COPD-related ailments than the non-diseased group. Moreover, athletes reported major physiological and psycho-social retardation trends upon return-to-sport fora from COVID-19 lay-off restrictions. Creating online digital coaching bionetwork websites could substantially enhance 'Work from Home' training environments. Digital health support synergies through virtual tele-health conferences and video/zoom forums could further provide practical resolutions for athletes' physiological and psycho-social parameters. Moderate to high intensity aerobic indoor training regimes could serve to attenuate retardation of quality skeletal tenacity and capacitate cardio-pulmonary activities from the effects of COVID-19 restrictions. SARSoriented athletes require appropriately designed training regimes that are within their motoric aptitudes to avoid compromising their cardiopulmonary-related ailments. This allows for gradual alterations of physiological body processes from the onset of training workouts.
\end{abstract}

Keywords: Cardiopulmonary loading, regime, SARS, coronavirus, Covid-19, musculoskeletal

\section{Introduction}

Physiological, psycho-social and emotional parameters are critical skill and technical performance motifs in sports endeavors. Despite their noteworthy basis for high-quality athletic efficiencies, training and psycho-social deficiencies are inevitable challenges for athletes in competitive locales. The large scale pandemic of COVID-19 has presented multiple health-related problems in players as restrictive measures forcibly exterminating them from organized sport settings. Subsequently, this pre-disposed them to sedentary lifestyles. The emergence of coronavirus (SARS-CoV-
2) has since prompted many governments to formulate legislative mechanisms to abate viral spread during spectatorship and sports training encounters. This orchestrated lasting impact on health outcomes in the quality of life with farreaching threats to public, socio-psychological and physiological health aspects in professional and nonprofessional sports. While these intransigencies have largely permeated into the normalcy of sporting and operational training routines for most sports boards, sports-inclined populations have ostensibly been thrown into a murky era of sedentary style of living. The breadth of global 
challenges and uncertainties need re-casting for sports scientists to embrace new emerging trends as a rescue basis for positive health and wellness for players. This study focused on the impact of COVID19 lockdown in view of cardiopulmonary loading among return-to-sport following long lay-offs from sport contexts. It explored on sport participation risks among players with Severe and non-Severe Respiratory ailments within training and exercise settings. The study was guided by the following research questions:

1. To what extent has COVID-19 lockdown impacted on players' participation in sport?

2. What challenges are encountered by returnto-sport COVID-19 affected players?

3. How far does training impact on players' cardiopulmonary parameters during exercise?

4. How can training be enhanced for COVID-19 affected players during lockdown period?

\section{Review of Related Literature}

This section presents the empirical review of literature related to the topic based on the 4 research questions that guided the study.

\section{COVID-19 lockdown and Sports participation}

The global epidemiological impact of COVID-19 on the morbidity levels has led WHO declaring it as a public health international catastrophe (MillanOnale et al., 2020). This unprecedented calamity has presented glaring effects on the sport and exercise industry, particularly on the professional and elite sport (Matthew, Hull, Rogers et al., 2020). While mass gatherings and sporting events inherently insulate human beings' depiction of social, symbolic embodied with culturally loaded meanings (Parnell, Widdop, Bond \& Wilson, 2020), suspension of adhoc and potentially super-spreader events have been effected. Further, burden-service gaps with high budgetary economic costs (Luiz, Junics, Saulo, Van Mechelen \& Verhagen, 2017) have prompted WHO and IOC to comprehensively invest in risk economic strategies, research promotion and scientific advances to help abate the devastating effects of coronavirus. Despite the pessimistic impact on professional elite sport, leisure and sport exercise (Matthew et al., 2020) uncertainties still hang on sports industry's full resuscitation and future with regard to athletes' neuromuscular, cardiovascular impairments and subsequent injury risks on return-to-sport (Sarto, Impellizzeri, Sporri et al., 2020). The likelihood of SARS-COV-2 endemic is inevitable due to emerging multiplicity of severe acute respiratory challenges. This has invariably seen Zimbabwean sport grinding to a halt.

The large-scale crisis of COVID-19 scourge has seen even developed countries like United Kingdom, German, America and South Africa s' economic, social and sports industries lurching under its affects (Ratten, 2020). No country has been spared from this historical catastrophe despite varied epidemiologic spread. Further, new highly transmissible variants of SARS-CoV-2 with a $70 \%$ severity rate has triggered pressure and spike of COVID-19 cases in UK, Brazil, South Africa and other parts of the world leading to stricter restrictions (Burki, 2021). SARS-Co-V2 is a novel zoonotic pathogen responsible for the cause of COVID-19 disease (Schellhorn et al., 2020) with resonance to the beta-corona virus that once ripped China in 2003. This highly infectious disease can potentially be spread through contact transmission (Wu et al., 2020). Consequently contact sports would not be spared as they are volatile grounds for the spread of the virus as most team sport rules are contact in nature.

\section{Psycho-sociological Impact of COVID-19} Coronavirus presents multi-socio-psychological outcomes on athletes. While lock down and selfquarantine may promote sedentary behaviors and severe disabling disorders (Lippi et al., 2020), emotional stressors of quarantine and training layoffs (Brooks, Webster et al., 2020) are inevitable traits. These uncertainties and breadth of COVID-19 shocks have inexorably disrupted normal operational sports training routines, triggering new trends of 'Work from Home' regimes (Kniffin, Narayanan, Anseel, et al., 2020). Subsequently, many Zimbabwean athletes have been isolated from supports network and normal training routines which are potential psychological management enhancers of stress, anxiety, frustration and depression. Zimbabwe has stepped from a 4 to 2 tier phase as risk-monitoring assessments on this epidemic with the Ministry of Sport suspending most contact sport leagues, let alone tertiary sport which has experienced total extinction for the past one and half years. For COVID-19 stricken athletes, regular check-ins and close contact with the technical team staff by way of tele-health consultations and video chats have been suggested to enhance athletes' psycho-social dimensions (Toredahl \& Asif, 2019). Consequently, the essence of inter-professional alliance and athlete-centredmedicine should be allowed in restorative settings 
to attenuate this burden-service gap of athletes' fractured physiological and socio-psychological resources (Dijkstra, 2018). Thus far, positive assurance from the social environment is critical in developing team homogeneity for athletes' hope pathways in sport to remain intact despite the unprecedented far-fetched COVID-19 effects.

\section{Training Challenges for Return-to-Sport Players during COVID-19 Lockdown}

Multiple challenges in sports training have been met. Nikolaids and Knechtle cited in Matus, Matus and Molino (2021) observed that Covid pandemic considerably changed all human activities allowing online videos/smart devices to replace sports and health sciences. Athletes engaged in exercises that were divorced from specialised physical activity monitoring. In another study of elite Cameroonian volleyball players, Guessogo et al. (2020) found out that lockdown restrictions adversely affected sports activities as players personally motivated themselves in the absence of expert supervision. These technical deficiencies led to training inadequacies with subsequent injury risks upon return-to competitive settings. Further, Mohr et al. (2020) reported Covid 19 extraordinary fitness and performance challenges for return-to-sport players, coaches, managers and clubs due to contact restrictions and solitude workouts. This drift necessitated training and rule adjustments for sports enthusiasts to fit in the 'new normal.' This subjugated sports persons to psychological mood irregularities, anxiety and frustrations regarding the future of sport.

Matus et al. s' (2021) multi-sport study of 42 Panamanian university athletes indicated a statistically significant difference of $90 \%$ motivation during pre-Covid-19 conditions than physical contact restrictions. Findings reflected technical and tactical gaps to be plugged as Covid-19 surge appear to exterminate the usual face-to-face physical proximity of technical operatives and players. Consequently attenuating the decay of endurance and neuromuscular-related performance in players (Silva, Brito, Akenhead and Nassis, 2016) needed to take centre stage. This could assist in reducing high sedentary behaviours (body mass and intramuscular body fat) induced by Covid-19 detraining effects.

\section{Cardiopulmonary Loading Parameters during Exercise/Training Settings}

Chronic Obstructive Pulmonary Disease (COPD) patients' (athletes) central hemo-dynamic responses to load can significantly be impaired by poor cardiac pathology and air way blockings. This has been reported to precipitate hyperinflation and dysfunctional breathing pressure variations with increased expiratory load (Laveneziana et al., 2014). In training and exercise settings COP adult-athletes risk severe hyperinflation and negative inspiratory intrathoracic pressures with greater risk of sustaining impairment in exercise-related workouts (Smith et al., 2019). They have been linked with greater cost of exercise than healthy persons due to elevated breathing costs and reduced locomotor mechanical inefficiency. Moderate loading implications on COPD patients (athletes) show greater reliance on marginal aerobic $\left(\mathrm{O}_{2)}\right.$ utilisations than healthy persons. Correlations between Inspiratory Capacity and Total Lung Capacity at matched aerobic exercise settings (Vo2) for COPD patients (athletes) show reduced volume, an impairment which is caused by elevated hyperinflation. Thus, significant hyperinflation has consequential loading impact on the cardiovascular system of COPD athletes during high intensity workouts (Smith et al., 2019). In consequence appropriate load dosages on athletes' cardiopulmonary capacities during potential latent phase are critical to avoid overload of return-to-sport athletes. Training dosages should allow for gradual recuperation and adaptation of damaged or atrophied tissues from low to high load cadence within preferred training frameworks. As Toresdahl \& Asif, (2019:10) rightly suggest, there is need 'to limit training sessions that is in tandem with aerobic threshold capacities to prevent COVID-19'.

Lockdown de-training effects from sedentary living for sports persons have precipitated multiple physiological and psychological repercussions for return-to-active sport players (Sarto et al., 2020). COVID-19 is a severe acute respiratory symptom that leads to Acute Respiratory Distress Syndrome connected with substantial respiratory challenges (Gupta, Madhavan, Landry et al., 2020). Its hyperinflammatory response intensifies the development of ARDS which compromises multiple extra pulmonary acute coronary processes, acute kidney injury, neurologic illnesses and irregular cardiac challenges (Madjid et al., 2020). Its high thoracic pressure mechanisms cause hypoxemic pulmonary vasoconstriction complications (Cui et al., 2020). This negatively impacts on the right ventricle and can lead to inflammatory atherosclerosis plague rapture with distal embolisation. COVID-19 causes 
endothelial cell damage, thrombo-inflammation and deregulation of the immune system leading to endothelialitis (damage of cardio-respiratory structures/organs, blood vessels). This inhibits fibrinolysis processes with subsequent loge of blood clots deposits which blocks blood vessels (Varga et al., 2020). Subsequently this impairs the lymphatic and alveoli routes. These variations regarding pro and anti-coagulant pathways precipitates heart, kidney and lung dysfunctions which ultimately leads to death (Gupta et al., 2020). These clinical presentations put players with SARS-related disorders at high risk of coronavirus effects during aerobic workouts escalating mortality rates.

COVID-19 is linked to biochemical myocyte necrosis and heightened cardio biomarkers of troponin or creatine causing acute exercise and subsequent death (Shii et al., 2020). For instance, Huang et al.'s (2019) study found out that $12 \%$ of patient-cases exhibited elevated levels of troponin indicating myocardial injury. Similarly, Shi et al. (2020) announced $8 \%$ acute cardiac injury from 6 studies involving 1527 patients. In another COVID-19 related study, $17 \%$ of patients were diagnosed of acute arrysthimias (Wang et al., 2019) suggesting that in sports cardiology spheres overload training can potentially lead to cardio-vascular overload (Dores et al., 2020). Presence of other virus infections and their replications can be elevated through high load intensities eventually prejudicing heart tissues to severe damages (Schellhorn et al., 2020) leading to increased mortality prevalence. Shii et al., (2020) reported myocardial damage and heightened cardiac biomarkers among 55\% of COVID-19-stricken patients with pre-existing cardiovascular diseases. Consequently they were at great risk of sustaining endothelialitis with heightened extra pulmonary loading on the expiratory and inspiratory pathways of the cardio-respiratory organs (Smith et al., 2018). Possible replications of this nature, to some extent, are most likely not to spare Zimbabwean athletes experiencing sedentary life-styles from COVID-19 restrictions.

Symptomatic effects of coronavirus can induce cardio-myopathology leading to dilated cardiac chambers and systolic impairment (Schellhorn et al., 2020). Such incapacitation can potentially rapture cardiac tissues causing myocarditis (Madjid et al., 2020) with high risk of cardiogenic shock (Clerkin et al., 2020). Thus, myocardial tissue resilience can be overwhelmed by high surge levels from incompatible arterial and venous pulmonary loading with stressful effects on the Stroke Volume and Heart Rate. High impact training loads, in addition to pre-existing corona virus damages, further predispose players to severe cardiovascular damages.

Load intensity is a critical biomarker of an athlete's cardiac output during training (Chyne et al., 2017). More negative intrathoracic pressures during inspiration and dynamic hyper-inflation have been linked to decreased Left Ventricle Stroke Volume which, in part could be due to direct ventricular interaction in healthy human at rest. Voluntary hyper-inflation increases stroke volume to control levels in exercise emanating from more negative breathing pressures (Stark-Leyva et al., 2004). Thoracic pressures on central hemo-dynamics can be affected by breathing mechanisms and pulmonary vascular alterations with subsequent impact on respiratory mechanics (Chyne et al., 2017). On the other hand, expiratory loading can reduce cardiac output and Stroke Volume. More negative intrathoracic pressures (per breath) during inspiration have direct positive links with cardiac output. Total Lung Capacity negatively correlates with systemic vascular resistance in COPD patients (athletes) during moderate and vigorous training (Smith et al., 2018). SARS-CoV-2 virus binds to ACE 2 (a receptor that serves many protective pathways in the lungs) found in the upper airways after protein spike on the virus is activated by transmembrane protease serine. Athletes with COPD-oriented ailments are most likely to be at risk of cardiac stress during exercise. Subsequently, this could cause functional decline and reduced exercise tolerance (Miller et al., 2021). Thus, knowledge of exercise demand and athletes' medical objective health profiles could serve as critical evaluative tools for sports clinicians during off-season, in-season and return-to-sport athletes.

\section{Enhancing training during COVID-19 Lockdown}

Physiological research on endurance exercise has been documented. High-Intensity Interval Training (HIIT) based on cardiac parameters of Stroke Volume and Cardiac Output (Falz et al., 2019) exists. Training modality diversities (Fisher et al., 2015) have reportedly been linked to varying load intensities, durations and interventional frequencies in relation to acute physiological and cardiopulmonary parameters of athletes (Green et al., 2017). Training and load intensities correlate with peak cardiopulmonary response especially higher aerobic consumption, Cardiac Output, breathing volume and Heart Rate during high 
intensity workouts. Falz et al.'s (2019) randomized crossover study substantiated that Interval Training and Moderate-Intensity Continuous Training exert greater acute cardiopulmonary effects than ST. Training impact time and intended targeted (HR) range critically determines athlete's exercise duration in the training impact zone for realisation of improved training target (Cornelis et al., 2016). Researches indicate that HIIT can significantly improve left ventricle ejection fraction and left ventricular end-diastolic diameter (Baekkerud et al., 2016) with improved heart pumping efficiency and cardiac output baseline level of aerobic capacity (Astorino et al., 2017). Isometric exercises potentially reduce SV (Taylor et al., 2017) which emanates from an increased acute left ventricular afterload and intrathoracic pressure (Smith et al., 2018). Subsequently, appropriately designed and prescribed training principals can better-off produce positive physiological benefits relative to athletes' capacity levels.

High Intensity Functional Training regimens have been popularised among fitness enthusiasts as a 'Top 10 Fitness Trend' in 2018 (Feito et al., 2018). Its functional multi-joint movement during aerobic and muscle strengthening exercises elicits universal motor recruitment patterns in multiple plannary movements which, in subsequence, improves cardiovascular endurance strength and flexibility (Poston et al., 2016; Heinrich et al., 2015). Unlike the unimodal nature of HIIT modes, HIFT have been linked to multi-modal-orientations inclined towards functional exercises. Thus, use of such training systems could be useful when re-orienting returnto-sport athletes following recovery from COVID-19, COPD related athletes. This improves their cardiovascular and cardiac output baseline levels of aerobic capacity (Astorino et al., 2018). Although such training regimens could potentially improve athletes' cardiovascular parameters, Falz et al. (2019) and Cornelis et al. (2016) argue that particular attention should also be paid to prescribed training impact zone load dosages. This evades overcapacitating and overwhelming of the cardiovascular system and recuperating myocardial tissues (Dores et al., 2020; Schellhorn et al., 2020) from hyper-inflation and intrathoracic pressures during exercise-related hemo-dynamics.

In consequence, appropriate load dosages on athletes' cardio-pulmonary capacities during potential latent phase are critical for coaches so that athletes on the verge of return-to-active cycle of participation (from long lay-off) need not be overloaded. Prescribed training dosages should allow for gradual recuperation and adaptation of damaged or atrophied tissues from low to high loading intensities that are in tandem with the adopted training regimes.

\section{The New Normal Operational Basis in Sport}

WHO's (2021) morbidity lens regarding COVID-19 scourge, necessitates sports scientists, sports federations, clubs and recreational to embrace training and exercise modifications. To this end, legislative courses of action have been effected (Futterman, Panja \& Keh, 2020) as preventive measures on this pandemic. In the wake of Covid-19 intransigencies, focus should be made on best service delivery approaches. The new normal should find its pathways through creation of digital coaching ecosystem, pedagogical competences and digital skill proficiencies (European Commission, 2021). In a recent study, Teodorescu et al. (2021) observed that $87.22 \%$ of Romanian coaches who used online training during first lockdown adjusted their initial training objectives. Of these $83.5 \%$ acquired IT tools through self-funding with $51.84 \%$ failing to engage all athletes in online training. Despite these upheavals, a clear trajectory and basis has been set in view of the new trends that should supercede traditional sports training and coaching approaches. While these challenges significantly vary with settings, technological advancements should be embraced as Covid-19 is likely to develop into an endemic feature in most sporting societies.

\section{Availing Physiological Return-To-Sport Readiness Tests}

From a public health and sport exercise setting, preparticipation medical screenings, historically, have a bearing that serve to identify athlete's impairments and activity limitations so that re-assessment allows health-service providers to formatively weigh-up current status and adapt interventions accordingly (Target et al., 2018). Sports-inclined clinicians and exercise therapists are able to arrive at informed decisions regarding institution of suitable exercise prescriptive tests (Zumana et al., 2019). Screening of cardiac, neuromuscular and pulmonary functions (Sullivan et al., 2019) for return-to-sport athletes following COVID-19 recovery should, therefore, take precedence, and be appropriately instituted through aerobic pulmonary functional tests and neurophysiological tests (Toole et al., 2019). Athletes with comorbid-oriented ailments and those recuperating from COVID-19 require clinical medical assessments 
prior to commencing Graduated Return-To-Play Protocol. Researchers aver that this GRTP progressively monitors parameters of cardiac output, respiration function, Resting Heart Rate, stress, fatigue through less aerobically intense sports (Baggish et al., 2020). Regularised checking of players' pathophysiological parameters regarding stroke volume, cardiac output and blood pressure should be regularised (Wilson et al., 2020) during transitional potential latent periods following recuperation from COVID-19.

Particular attention should be given to appropriate load intensity and load tolerance to cardiopulmonary/cardiovascular loading structure to avoid re-ignition of recuperating cardiac and musculotendinous synchronization following recovery from COVID-19 effects. Additionally, WHO Covid-19 testing and preventive protocols for players and management team should be periodically done by health service providers. Treatment and self-isolation measures should be encouraged and appropriately carried out.

\section{Research Methodology}

In this section the research methodology used during the study is presented.

\section{Research Design}

The study employed the descriptive, prospective cohort design anchored on the quantitative approach.

\section{Population and sampling}

The study population was 200 participants from which a sample of 25 coaches and 50 players from selected tertiary institution teams in Masvingo Province were drawn to fill the questionnaire. Study participants were drawn using the stratified random sampling. A convenient size for the study was determined through Taro Yamani's (1970) formula. Bowley's proportional allocation formula was then used to statistically allocate participants into their particular strata.

\section{Validity and Reliability}

Cronbach alpha statistics was used to determine the internal consistency of the questionnaire items before the instrument was used in the field. The test yielded the Cronbach's Alpha of 0.8 which indicates acceptable reliability. The instrument was pilottested using a smaller related sample prior to its administration to the intended respondents

\section{Statistical Treatment of Data}

Collected data was statistically presented on tables through frequencies and percents.

\section{Ethical considerations}

Ethical clearance was sought from the Universities and Teachers Colleges Authorities. Informed consent was sought from study participants drawn from the selected institutions. Anonymity and Confidentiality of data were established to meet ethical standards.

\section{Results and Discussion}

This section makes a presentation of the results for the study basing on the 4 research questions that guided the study:

Research question 1: To what extent has lockdown impacted on sports training programs?

Table1 results substantiate moderate (24\%) to major $(60 \%)$ effects of lockdown trends on planned training regimes with no training/competition modifications (72\%). Multiple training regimes have been popularized (Dores et al., 2020; Schellhorn at al., 2020; Faltz et al., 2019; Smith et al., 2018; Feito et al., 2018; Astorino et al., 2018; Green et al., 2017). For Return-To-Sport COVID-19 recuperating athletes, prescribed training exercises chosen could substantially eliminate venous thrombolysis debris. Training that raises the Heart Rate promotes vasodilation that potentially sweep formed thrombi debris. This improves blood flow/Stroke Volume and myocardial pumping efficiency that boosts mitochondrial activity allowing for transportation of energy elements to the working muscles during low to high intensity workouts. Huang et al. (2020) and Shii et al. s' (2019) studies found correlations between COVID-19 with myocyte necrosis and heightened biomarkers of troponin or creative which instigates acute exercise.

Table 1: Modified training Coaches' Questionnaire $(\mathrm{N}=\mathbf{2 5})$

\begin{tabular}{lcccc}
\hline Parameter/Attribute & No effect & Minor & Moderate & Major \\
\hline Any training modifications? & $18(72 \%)$ & $2(8 \%)$ & $3(12 \%)$ & $2(8 \%)$ \\
Lockdown impact on planned training schedules? & $1(4 \%)$ & $3(12 \%)$ & $6(24 \%)$ & $15(60 \%)$ \\
\hline
\end{tabular}

Further, studies confirm elevated effects of troponin of myocardial infarction and acute arrysthimias.
From a sports cardiology perspective overload training can prospectively subjugate 
pathophysiological routes through extra pulmonary loading on player's expiratory and inspiratory pathways. This develops high risks especially on players with pre-existing comorbid-oriented ailments (Gupta et al., 2020; Dores et al., 2020; Schellhorn et al., 2020). Turning on to this study, however, extra care should be given to athletes recuperating from COVID-19 long lay-off effects on load tolerance, intensity, and pulmonary resilience during high impact workouts. This protects their aerobic and anaerobic parameters from high surge levels of incompatible arterial and venous stresses. In this regard, embracing serialized multi-modal scientific monitoring tests that address athletes' cardiopulmonary and cardiovascular parameters could episodically be carried out for recuperating and asymptomatic COVI-19 athletes. As Silva et al. (2016) rightly puts it, focus should be on attenuating the decay of endurance and neuromuscular-related performances in players.

Table 2: Participation: Coaches n (25)

\begin{tabular}{lcccc}
\hline \multicolumn{1}{c}{ Parameter } & No effect & Minor & Moderate & Major \\
\hline Extent of challenges experienced? & - & $2(8 \%)$ & $3(12 \%)$ & $20(80 \%)$ \\
Impact on players' psychological resources? & - & $2(8 \%)$ & $8(32 \%)$ & $15(60 \%)$ \\
\hline
\end{tabular}

Table 3: Player's performances: Coaches' responses $\mathrm{n}$ (25)

\begin{tabular}{lcccc}
\hline \multicolumn{1}{c}{ Parameter } & No effect & Minor & Moderate & Major \\
\hline Players' physiological retardations during lock down? & - & - & $2(8 \%))$ & $23(92 \%)$ \\
Adherence levels to training regimes? If any? & $2(8 \%)$ & $8(32 \%)$ & $6(24 \%)$ & $9(36 \%)$ \\
Satisfied with training achievement to your players? & $20(80 \%)$ & $2(8 \%)$ & $2(8 \%)$ & $1(4 \%)$ \\
\hline
\end{tabular}

Table 4: Participation during lockdown: Players $\mathrm{n}(50)$

\begin{tabular}{lcccc}
\hline \multicolumn{1}{c}{ Parameter/Attribute } & No effect & Minor & Moderate & Major \\
\hline Extent of participating in training regimes? & $29(58 \%)$ & $5(10 \%)$ & $7(14 \%)$ & $9(18 \%)$ \\
Rate the extent of challenges you experienced on your & - & - & $2(4 \%)$ & $2(4 \%)$ \\
training sessions & & & & $48(96 \%)$ \\
\hline
\end{tabular}

Table 5: Training/Competition Modifications: Players $\mathbf{n}(50)$

\begin{tabular}{|c|c|c|c|c|}
\hline Parameter/Attribute & No effect & Minor & Moderate & Major \\
\hline Any modified training competitions received from coaches? & $33(66 \%)$ & $8(16 \%)$ & $6(12 \%)$ & $3(6 \%)$ \\
\hline Extent of load intensities? & $35(70 \%)$ & $5(10 \%)$ & $4(8 \%)$ & $6(12 \%)$ \\
\hline Training satisfaction? & $42(84 \%)$ & $2(4 \%)$ & $4(8 \%)$ & $2(4 \%)$ \\
\hline
\end{tabular}

As shown on Table 2, lockdown presented major participation and training challenges (80\%) that impacted players' psychological resources (92\%) with only a few minor cases (16\%). Whereas only a segment of players and coaches (Tables 1 and 2) revealed full engagement in modified trainings with minimal challenges, the majority remained much isolated from favorable environments due to COVID19 restrictions. Consequently, their physiological, emotional and psycho-social instincts remained subdued and incapacitated for the entire part of lockdown, to date. As averred by Lippi et al. (2020) and Sarto et al. (2020), lock down encouraged detraining effects and sedentary behaviors with hindrances to most sporting populations in Zimbabwe. Although this could be viewed as an enabling factor for only a minority of well-resourced people, Kniffin et al. (2020) however, bemoans on the uncertainties and breadth of COVID-19 shock which, certainly diverted normal training tendencies towards more of new innovative trends of Work From Home (WFH) etiquette. This new structure is most likely to trigger multi-upheavals before sportsinclined persons get necessary acquaintances.

According to Table 3, psychological traumas of COVID-19 led to physiological retardations (92\%) which was way below coaches' training expectation levels (80\%). Thus players risked injury sustenance from low levels of training intensities.

Table 4 results indicate that the majority of players experienced major challenges during the lockdown (96\%) despite varied participation extends (58\%, no effect; $18 \%$, major, $14 \%$, moderate and $10 \%$, minor).

Table 5 indicates that training adjustments were viewed differently by coaches: $66 \%$ considered them as having no effect, $16 \%$ claimed training at minor, $12 \%$ moderately achieved while $6 \%$ indicated 
having scored major achievements. Lock down had drastic implications on load intensities $(70 \%, 12 \%$, $10 \%, 8 \%)$ and training satisfaction levels ( $84 \%$ no effect $8 \%$, moderate and $4 \%$ apiece for major and minor). It is in this sense that Teodorescu et al. (2021) found out that $51.84 \%$ of Cameroonian coaches failed to engage players in online training sessions.
Regarding performance rating levels, players could have been affected by different levels of 'support synergies' received during lockdown (Table 6). Of note, the greatest segment received no support (54\%) with $18 \%$ showing moderate support, $12 \%$ with support and $8 \%$ having the least support. Variations depict levels of significance regarding the need to adhere to individualized training regimes in order to keep players in sound physiological states.

Table 6: Performance Ratings in lockdown: Players n (50))

\begin{tabular}{lcccc}
\hline \multicolumn{1}{c}{ Parameter/Attribute } & No effect & Minor & Moderate & Major \\
\hline Current fitness and performance levels rating? & - & $7(14 \%)$ & $12(24 \%)$ & $31(62 \%)$ \\
Extent of social support services from coaches and family? & $27(54 \%)$ & $8(16 \%)$ & $9(18 \%)$ & $6(12 \%)$ \\
\hline
\end{tabular}

Table 7: Psychological lockdown impact: Coaches n (25)

\begin{tabular}{lcccc}
\hline \multicolumn{1}{c}{ Parameter } & No effect & Minor & Moderate & Major \\
\hline Disruption of players' life patterns? & - & $1(4 \%)$ & $5(20 \%)$ & $19(76 \%)$ \\
& & $7(28 \%)$ & $10(40 \%)$ & $8(32 \%)$ \\
Players' kinesiophobia injury worries on full return-to & & & & \\
participation? & - & & & \\
Emotional afflictions? & $2(8 \%)$ & $4(16 \%)$ & $8(32 \%)$ & $9(36 \%)$ \\
Isolation worries from normal training settings? & $3(8 \%)$ & $3(12 \%)$ & $9(36 \%)$ & $11(44 \%)$ \\
Loss of self-identity to team homogeneity? & & $5(20 \%)$ & $7(28 \%)$ & $10(40 \%)$ \\
\hline
\end{tabular}

Table 8: Psychological lockdown impact: Players n (50)

\begin{tabular}{lcccc}
\hline \multicolumn{1}{c}{ Parameter/Attribute } & No effect & Minor & Moderate & Major \\
\hline Level of psychological impact? & $2(4 \%)$ & $9(18 \%)$ & $10(20 \%)$ & $29(58 \%)$ \\
Level of emotional worry? & - & $2(4 \%)$ & $5(10 \%)$ & $43(86 \%)$ \\
Loss of self-identity and team homogeneity? & - & $5(10 \%)$ & $14(22 \%)$ & $31(62 \%)$ \\
Feelings of insecurity from COVID-19 impact? & - & $2(4 \%)$ & $3(6 \%)$ & $45(90 \%)$ \\
Any disruption of life patterns? & - & - & $2(4 \%)$ & $48(96 \%)$ \\
\hline
\end{tabular}

Research question 2: How far has COVID-19 impacted on players' participation in sports?

Results in table 7 explicates multiple psychological impacts with major disruptions of players' life patterns (76\%), loss of opportunity to present their self-identities $(40 \%)$ and isolation worries from normal training and team settings (44\%). More over emotional turmoil (68\%) further exhibited players' self-presentational kinesiophobia statuses (72\%) regarding low physiological incapacities upon their return-to-sport. This is in spite of a small section of coaches, who reported less anxiety levels on players' return-to-sport contexts (28\%), emotional afflictions (16\%), isolation worries (12\%) and loss of team identity settings (20\%). This suggests that such few players are involved in meaningful training regimes in home settings compared to the majority who happen to be in deprived locales.
As indicated in Table 8, high psychological spikes (58\%, $20 \%$, and $18 \%$ ) further substantiate severe costs on players' emotional resources $(86 \%)$, selfidentities (62\%, 22\%), insecurity (92\%) and disruption of 'personal and sport life patterns' (96\%). This could suggest a possible dead end to career routes for some in view of the emerging coronavirus endemic trends. Matus et al. s' (2021) multi-sport study of Panamanian university athletes revealed $90 \%$ pre-COVID-19 motivational trends compared to current physical contact restrictions. These findings are further substantiated by results in this study as players and coaches invariably experienced multiple psychological resources (Tables 7 and 8). As averred by Brooks et al. (2020), COVID-19 scourge precipitated emotional stressors of self-isolation, training lay-off frustrations and boredom. This corroborates well with findings of this study which reported $80 \%$ isolation worries 
from normal training among coaches and emotional worries/afflictions $(86 \%$ and $68 \%$ for players and coaches respectively).

Results in Table 9 further validate variations in the epidemiological spread of the coronavirus pandemic as depicted by wide-ranging frequencies of active and death cases reported. These geographical surge project high risks associated with public sport contact.
Pertaining to epidemiological spread of COVD-19 the presence of active $(44 \%, 18 \%)$ and death cases $(24 \%, 22 \%)$ in participants' areas of residence significantly varied but additionally exacerbated this turmoil (Tables 9 and 10). Varied epidemiological breadth could be attributed to degrees to which residents adhered to WHO preventive, testing and treatment protocols.

Table 9: Epidemiological spread of COVID-19: Coaches $\mathbf{n}(25)$

\begin{tabular}{lllll}
\hline Parameter & No effect & Minor & Moderate & Major \\
\hline Active cases reports in your area? & $1(4 \%)$ & $4(16 \%)$ & $9(36 \%)$ & $11(44 \%)$ \\
Death Epidemiological frequency reports in your area? & - & $5(20 \%)$ & $6(24 \%)$ & $14(56 \%)$ \\
\hline
\end{tabular}

Table 10: Epidemiological spread of COVID-19: Player $n(50)$

\begin{tabular}{|c|c|c|c|c|}
\hline Parameter/Attribute & No effect & Minor & Moderate & Major \\
\hline Active cases reports in your area? & $2(4 \%)$ & $26(52 \%)$ & $13(26 \%)$ & $9(18 \%)$ \\
\hline $\begin{array}{l}\text { Death Epidemiological spread of frequency reports in } \\
\text { your area? }\end{array}$ & $3(6 \%)$ & $33(66 \%)$ & $11(22 \%)$ & $3(6 \%)$ \\
\hline
\end{tabular}

Table 11: Health, symptomatic and preventive measures for players $n(50)$

\begin{tabular}{lcccc}
\hline \multicolumn{1}{c}{ Parameter/Attribute } & No effect & Minor & Moderate & Major \\
\hline $\begin{array}{l}\text { Frequencies of pre-participation medical examination } \\
\text { tests done? }\end{array}$ & $36(72 \%)$ & $7(14 \%)$ & $4(8 \%)$ & $3(6 \%)$ \\
Prior cardiorespiratory/cardiovascular related ailments? & $26(52 \%)$ & $13(26 \%)$ & $6(12 \%)$ & $5(10 \%)$ \\
Diagnoses of COVID-19 symptomatic signs? & $42(84 \%)$ & $5(10 \%)$ & $3(6 \%)$ & - \\
Adherence to WHO testing and preventive measures to & -- & - & $7(14 \%)$ & $43(86 \%)$ \\
COVID-19 during lock down? & & & & \\
\hline
\end{tabular}

Table 12: Health, symptomatic, and preventive measures for Coaches $\mathbf{n}$ (25)

\begin{tabular}{lcccc}
\hline \multicolumn{1}{c}{ Parameter } & No effect & Minor & Moderate & Major \\
\hline Pre-participation medical examination tests frequency? & $19(76 \%)$ & $3(12 \%)$ & $2(8 \%)$ & $1(4 \%)$ \\
$\begin{array}{l}\text { Any Health challenges/complaints on players' } \\
\text { physiological incapacities? }\end{array}$ & $2(8 \%)$ & $16(64 \%)$ & $3(12 \%)$ & $4(16 \%)$ \\
$\begin{array}{l}\text { Adherence to WHO testing/preventive measures to } \\
\text { COVID-19? }\end{array}$ & $3(12 \%)$ & $4(16 \%)$ & $5(20 \%)$ & $13(52 \%)$ \\
\begin{tabular}{l} 
Cardiopulmonary loading challenges during exercise? \\
\hline
\end{tabular} & $5(25 \%)$ & $4(16 \%)$ & $6(24 \%)$ & $10(40 \%)$ \\
\hline
\end{tabular}

Research question 3: How far does training impact on players' cardiopulmonary parameters during exercise?

This research question was responded to by players and coaches to indicate how far players' cardiopulmonary parameters during exercise.

Diverse responses to players' cardiovascular related ailments ranged from minor (26\%), moderate (12\%) to major $(10 \%)$ the training impact on with the greatest number not having experienced ailments of this nature (52\%). Most players revealed no COVID19 symptomatic signs while a total of $16 \%$ appeared symptomatic suggesting high susceptibility intensities on their pre-existing cardiovascularrelated ailments. Results further confirm high adherence levels to WHO COVID-19 testing and preventive protocols as shown by the figures of $86 \%$ and $14 \%$ respectively. Surprisingly, low awareness levels on the criticality of pre-participation medical examinations among players exist as confirmed from the larger section not involved in exercises of this nature $(72 \%)$. This makes them more susceptible to debilitating effects of coronavirus and cardiopulmonary loading risks. 
To a larger extent pre-participation return-to-sport medical readiness tests for players is not a highly esteemed exercise among most coaches (76\%) except for a few who see the significance attested to it (Table 12). This elucidates high risk levels during participation especially players with Severe Acute Respiratory-Oriented Pulmonary ailments. Despite low ratings of pre-participation medical screenings, WHO preventive measures however, were being adhered to (52\%-major; $20 \%$-moderate and $16 \%$ minor).

Pre-participation physiological readiness tests (medical) are an integral exercise priory carried out to determine and address any physiological defects a player might be subjected to in high intensity workouts. Only a few players are involved in medical examination exercises, possibly from well-resourced family support synergies. Although Sullivan et al. (2019) and Target et al. (2018) highly stress on the criticality of identifying players' physiological impairments, activity limitations as a formative way of weighing-up their current status, results of this study are at variance from these recommendations. Nevertheless, addressing players' physiological capacities forms the basis upon which good technical skills are founded. Based on findings of this study coaches were not adhering to preparticipation examination protocols. This possibly implies that they indulged in ad hoc training regimes which did not fully address players' neurophysiological, pulmonary functions, cardiovascular parameters as well as comorbid-oriented aliments. Thus far, results are not in confirmation with advances made in earlier studies (Baggish et al., 2020; Toresdahl \& Asif, 2020; Toole et al., 2019).

Research question 4: How can training be enhanced for COVID-19 affected players during lockdown period?

In view of the span of Covid 19 impact regarding findings from the aforementioned sections, this scourge is in tandem with Mohr et al. (2020) who observed Covid-19 extraordinary fitness and performance challenges for return-to-sport players and coaches. Major emerging sources were contact restrictions and unsupervised solitude training engagements. Similarly, Lippi et al. (2020) rightly admits that lockdown promoted sedentary behaviors with severe disabling disorders. For instance, risks of developing chronic coronaryrelated ailments. This is in sync with findings of this study. Although Teodorescu et al. (2021), Nikolaids and Knechtle (in Matus et al., 2021) contemplate embracing of online digital coaching ecosystems, training modification adjustments to this approach for coaches in this study were problematic $(60 \%)$. This is further reflected in players' physical participation challenges $(80 \%)$ probably due to insufficiency of IT tools, digital skill deficiencies and poor internet connectivity.

\section{Conclusions and Recommendations Conclusions}

Emerging findings revealed substantial disruption of training normalcy from lockdown restrictions as contact sport faced total extinction. Players engaged in solitude and unsupervised training sessions which predisposed them to injury risks. In addition, high cardiopulmonary loading intensities compromised health conditions of athletes with COPD-related ailments than the non-diseased group. Furthermore, athletes reported major physiological and psychosocial retardation trends upon return-to-sport fora from COVID-19 lay-off restrictions.

\section{Recommendations}

Creating online digital coaching bionetwork websites could substantially enhance 'Work From Home' training environments. Digital health support synergies through virtual tele-health conferences and video/zoom forums could further provide practical resolutions on athletes' physiological and psycho-social parameters. Moderate and high intensity aerobic indoor training regimes could serve to attenuate retardation of quality skeletal tenacity and capacitate cardio-pulmonary activities from the effects of COVID-19 restrictions. SARS-oriented athletes require appropriately designed training regimes that are within their motoric aptitudes to avoid compromising their cardiopulmonary-related ailments. This allows for gradual physiological alterations of body processes to be made from the onset of training workouts.

\section{References}

Astorino, T.A., Edmunds, R. M., Clark, A., King, L., Gallant, R. A., Namm, S., \& Wood, K. M. (2017), High intensity interval training increases cardiac output and VO2 maximal, Medicine Science Sport and Exercise, 49: 265-273.

Barbian, E., Agostini, D., Polidori, E., Potenza, L., et al., (2015), The Pleitropic Effect of Physical Exercise on Mitochondrial Dynamics in Aging Skeletal Muscle, Oxidative Medicine 
and Cellular Longevity Volume 2015, http://dx.doi.org/10.1155/2015/917085.

Baekkerud, F. H., Solbery, F., Leinon, I. M., Wisloff, U., Karlsen, T., \& Raogmmo, O. (2016), Comparison of three popular exercise modalities on VO2 maximal in overweight and obese, Medicine Science Sports Exercise, 48: 491-498.

Baggish, A., Drezner, J. A., Kui, J., Martinaz, M., \& Pruktin, J. M. (2020), Resurgence of sport in the wake of COVID-19: Cardiac considerations in competitive athletes, British Journal of Sports Medicine, 54 (19): 6-12.

Brooks, S, K., Webster, R, K., Smith, L, E., Woodland, L., Wessley, S., Greenberg., et al., (2020). The psychological impact of quarantine and how to reduce it: rapid review of the evidence. Doi: https://doi.org/10. 1016 /SO 140-6736.

Burki, T., (2021), Understanding variants of SARSCOV-2, The Lancet, World Report, 397 (10273):462.

Chyne, W. S., Williams, A. M., Harper, M. I., and Eves, N. D. (2017), Acute volume loading exacerbates direct ventricular interaction in a model of COPD, Journal of Applied Physiology, 123: 1110-1117.

Clerkin, K. J., et al., (2020), Coronavirus disease 2020 (COVID-19), and cardiovascular diseases, Circulation, 141: 1648-1655.

Cornelis, G., Leinon, M., and Kui, J. (2016). Cardiorespiratory challenges among COPD patients, Sports Medicine, 16 (6): 24-30.

Cui, S., Chen, S., Li, X., Liu, S., \& Wang, F. (2020), Prevalence of venous thrombo-embolism in patients with severe novel coronavirus pneumonia, Journal of Thrombosis Haemostats. 18: 1471-1424.

Dijkstra, H. P., Pollock, N., Chakraverty, R., and Alonso, J. M. (2018). Managing the health of the athlete: a new integrated performance health management and coaching model. British Journal of Sports Medicine, 48, (7): 1-17.

Dores, H., \& Cardin, N. (2020), Return-To-Play after COVID-19: a sport cardiologist's view, British Journal of Sports Medicine, 0 (0): 1-2.
European Commission, Digital Education Action Plan (2021-2027), Resetting Educational Training for the Digital Age, (2021). https:// 4c.europa.eu/education.

Falz, H, M., Mohr, C., and Dores, H. (2019). Sport participation in lockdown, Exercise Medicine Science, 7: 69-74.

Feito, Y., Heinrich, K. M., Butcher, C. J., and Piston, W. S. E. (2018), High-Intensity Functional Training (HIFT): Definition and Research Implications for Improved Fitness. Sports, 6 (76): 3-19.

Fisher, G., Brown, M. M. B., Arlcon, A., Noles, C., Winhood, L., Resueler, H., George, B., Jeansonne, M. M., \& Alison, D. B. (2015), High intensity interval exercise versus moderate-intensity-training for improving cardio metabolic health in overweight or obese males: a randomised control trial. PLOS One, 10:20138853.

Futterman, M., Panja, T., and Keh, A. (2020). As coronavirus spreads, Olympic face ticking clock and a tough call, New York Times, https://www.nytimes.com.

Guessogo, W, R., Bika-Lele, E, C., Mban-Brian, W., Mandjek, A. R. D., Temfemo, A., Mandengue, S. H., and Assomo-Ndemba, P. B. (2020). Semi-Lockdown on Sports and Physical Activity Behaviours of Cameroonian Elite Volleyball Players: A Cross-Sectional Study, Electronic Physician, 13 (1), 78137821.

Green, N., Wertz, T., Laporta, Z., Mora, A., Serbes, J., \& Astorino, T. A. (2017), Comparison of acute physiological and psychological responses between moderate intensity continuous exercise and 3 regimes of high intensity training, Journal of Strength Conditioning Res. https://doi.org /10.1519/jsc.

Gupta, A., Madhavan, M. V., Sehgal, K., et al., (2020), Extra pulmonary manifestations of COVID-19, Nature Medicine, 26: 1017-1032.

Huang, C., Wang, Y., Li, X., et al., (2020), Clinical features of patients infected with 2019 novel corona virus in Wuhan, China, The Lancet, 395: 492-506. 
Heinrich, K. M., Becker, C., Carlisle, T., Gilmore, K., Hanser, J., Frye, J., and Harms, C. A. (2015), High intensity functional training in power functional movement and body composition among cancer survivors: A pilot study, European Journal of Cancer Care, 24: 812817.

Laveneziana, P., Webb, K. A., Wadell, K., Neder, J. A., and O'Donnell, D. E. (2014), Does expiratory muscle activity influence dynamic hyperinflation and extertional dyspnoea in COPD? Respiratory Physiology Neurobiology, 199: 24-33.

Lippi, G., and Piebanl, M., (2020). Laboratory abnormalities in patients with COVID-2019 infection, Clin.Chem. Lab. Med, 58 (7): 11311134.

Luiz, C, H. J., Saulo, D, B., Van Mechelen, W., and Verhagen, E. (2017), Measuring sports injuries on the pitch: a guide to use in practice, Brazil Journal of Physical Therapy, 19 (5), https://dx.doi.org/10.1590/bjpt-rbf. 2014.0110.

Madjid, M., Safari-Naeni, P., Solomon, S. D., et al., (2020), Potential effects of corona viruses on the cardio-vascular system: a systematic review JAMA/Cardiol.doi.10.1001/jam acar dio.2020.1286.

Matthew, G. W., Hull, J. M., Rogers, J., et al., (2020), Cardio-respiratory considerations for Return-To-Play in elite athletes after COVID19 infection: a practical guide for sport and exercise medics and physicians, British Journal of Sports Medicine, 54: 1157-1161.

Millan-Onate, J., Rodriguez-Morales, A. J., CamachoMoreno, G., Mendoza-Ramirez, H., Rodriguez-Sabogal, I. A., \& Alvarez-Moreno, C. (2020), Anew emerging zoonotic virus of concern: the 2019 novel Coronavirus (COVID-19), Infectio, 24 (3), doi:10.22354/in v24i3.848.

Miller, N. L., Silbernagel, K. G., Thorborg, K., et al., (2021), Tendinopathy, Disease Primers, I D: (2021):7.

Mohr, M., Nassis, g. P., Brito, J., Randers, M. B., Castagno, C., Parnell, D., and Krustrup, G. (2020). Return to football after Covid-19 lockdown: Managing sports and leisure, https://doi.org.10.1080/23750472.2020.
Parnell, D., Widdop, P., Bond, A., \& Wilson, R. (2020), COVID-19 networks \& sport, British Journal of Sports Medicine, 54: 117-118.

Poston, W. S., Haddock, C. K., Heinrich, K. M., Jahnke, S. A., Jitrarin, N., \& Batchelor, D. B. (2016), Is high intensity functional training (HIFT) Crossfit safe for military fitness training? Military Medicine, 181: 627-637.

Ratten, V. (2020), Coronavirus entrepreneurship, https://www.emarald.com/insight/13552554.htm.

Schellhorn, P., Klingel, K., \& Burgstahler, C. (2020), Return-To-Sport after COVID-19 infection: Do we have to worry about myocarditis? Oxford University Press, Public Health Emergency Collection.

Sarto, F., Imperillezi, F. M., Sporri, J., Porcelli, S.., et al., (2020), Impact of potential physiological changes due to COVID-19 home confinement on athlete health protection in elite sports: a call for awareness in sports programming, Retrieved from osf.10/ pre prints/sport xiv4z63a.

Shii, S., Qin, M., Shen, B et al., (2020), Cardiac injury on patients with corona virus disease, JAMA Cardiology, 2020.

Silva, J. R., Brito, J., Akenhead, A., and Nassis, G. P. (2016). The transition period in soccer: A window of opportunity, Sports Medicine, 46 (3): 305-313.

Smith, J. R., Johnson, B. D., \& Olsen, T. P. (2019), Impaired central hemodynamics in chronic obstructive pulmonary disease during submaximal exercise, Journal of Applied Physiology, 127: 3-6.

Stark-Leyva, K. N., Beck, K. C., \& Johnson, B. D. (2004), Influence of expiratory loading and hyper-inflation on cardiac output during exercise, Journal of Applied Physiology, 96: 1920-1927.

Sullivan, S, B, O., Schmitz, T, J., and Fulk, G. (2019), Physical Rehabilitation, $9^{\text {th }}$ Ed, Davis Company, USA.

Targett, S., Bere, T., and Bahr, R. (2018). The Role of Pre-Participation Assessment (PPA) and screening in Handball. Handball Sports Medicine. pp: 115-124. 
Taylor, K. A., Wiles, J. D., Coleman, D. D., Sharma, R., \& Odriscall, J. M. (2017), Continuous cardiatic autonomic and hemodynamic responses to isometric exercise, Medicine, Science Sports and Exercise, 49: 1511-1519.

Teodorescu, S., Bota, A., Popescu, V., Mezei, M., and Urzola, C. (2021). Sports Training During Covid-19 First Lockdown: A Romanian Coaches' Experience. https://doi.or g/1 0.3390 .

Toole, A. R., Ithurburn, M. P., Rauh, M. J., Hewett, T. E., Parteno, M. V., and Schmitt, L.C. (2019). Young athletes cleared for sports participation after Anterior Cruciate Ligament reconstruction: How many actually meet recommended return to sports criterion cut-offs? Journal of Orthopaedic and Sports Physical Therapy. 47 (11): 825-883.

Varga, A., et al., (2020), Endothelial cell infection and endothelialitis in COVID-19 patients, Lancet, 395: 1417-1418.
Yeo, T. J. (2020), Sport and exercise during and beyond the COVID-19 pandemic, The European Society of Cardiology, 0 (0): 1-3.

Wang, D., Hu, B., Hu, C., et al., (2019), Clinical characteristics of 138 hospitalised patients with 2019 corona viruses infected pneumonia in Wuhan, China, JAMA, 323:1061-1069.

Wu, Y. C., Chen, C. H., \& Chan, Y. J. (2020), The outbreak of COVID-19: An overview, Journal of Chinese Medical Association, 83 (3): 217220.

Zumana, N., Olivier, B., Godlwana, L., and Martin, C. (2019). Intra-rater and Inter-rater reliability of six musculoskeletal pre-participatory screening tests. South African Journal of

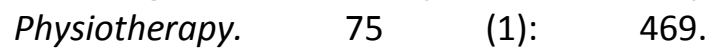
Doi.10.4102/saipv75i1.469.

World Health Organisation, (2021), COVID-19 Weekly Epidemiological Update, Edition 41, 25 May 2021. 